Tohoku Math. J

55 (2003), 477-485

\title{
SUR LE PROLONGEMENT ANALYTIQUE DE LA \\ SOLUTION DU PROBLÈME DE CAUCHY POUR \\ CERTAINS OPÉRATEURS DIFFÉRENTIELS DE PARTIE PRINCIPALE À COEFFICIENTS POLYNOMIAUX
}

Dédié à Professeur Norio Shimakura

\author{
YÛSAKU HAMADA
}

(Received September 4, 2001, revised July 26, 2002)

\begin{abstract}
In our preceding articles, by applying the results of Bieberbach, Fatou and Picard, we have constructed an example such that the domain of holomorphy of the solution admits an exterior point. Next we have studied the domain of holomorphy of the solution for differential operators with polynomial coefficients that concern the differential equations of Darboux-Halphen, of Chazy and of the modular function. In this article, we make some studies on the analytic continuation of the solution of the Cauchy problem for certain differential operators of principal part with polynomial coefficients.

Résumé. Dans nos articles précédents, en appliquant les résultats de Bieberbach, Fatou et Picard, nous avons construit un exemple tel que le domaine d'holomorphie de la solution ademette un point extérieur. Puis nous avons étudié le domaine d'holomorphie de la solution pour les opérateurs différentiels à coefficients polynomiaux, qui concernent les équations différentielles de Darboux-Halphen, de Chazy et de la fonction modulaire. Dans cet article, nous donnons quelques études sur le prolongement analytique de la solution du problème de Cauchy pour certains opérateurs différentiels de partie principale à coefficients polynomiaux.
\end{abstract}

Introduction. Leray [L] et Gårding, Kotake et Leray [GKL] ont étudié les singularités et un prolongement analytique de la solution du problème de Cauchy dans le domaine complexe. [P], [PW] et [HLT] ont étudié le cas des coefficients de fonctions entièrs.

Dans cet article, nous donnons quelques études des prolongements analytiques du problème de Cauchy pour certains opérateurs différentiels de partie principale à coefficients polynomiaux.

1. Notations et rappel de quelques résultats. Soit $x=\left(x_{0}, x^{\prime}\right)\left[x^{\prime}=\left(x_{1}, x^{\prime \prime}\right)\right.$, $\left.x^{\prime \prime}=\left(x_{2}, \ldots, x_{n}\right)\right]$ un point de $C^{n+1}$. On considère un opérateur différentiel d'ordre $m$, à coefficients de fonctions entières sur $\boldsymbol{C}^{n+1}$;

$$
a(x, D)=\sum_{|\alpha| \leq m} a_{\alpha}(x) D^{\alpha}, \quad D_{i}=\partial / \partial x_{i}, \quad 0 \leq i \leq n .
$$

Sa partie principale est notée $g(x, D)$ et supposons $g(x ; 1,0, \ldots, 0)=1 \operatorname{sur} \boldsymbol{C}^{n+1}$.

2000 Mathematics Subject Classification. Primary 35A20; Secondary 34A20.

Key words and phrases. Cauchy problem, analytic continuations. 
Soit $S$ l'hyperplan $x_{0}=0$, donc non caractéristique pour $g$. Étudions le problème de Cauchy

$$
a(x, D) u(x)=v(x), \quad D_{0}^{k} u\left(0, x^{\prime}\right)=w_{k}\left(x^{\prime}\right), \quad 0 \leq k \leq m-1,
$$

où $v(x), w_{k}\left(x^{\prime}\right)$ sont des fonctions entières sur $\boldsymbol{C}^{n+1}$ et $\boldsymbol{C}^{n}$ respectivement. Le théorème de Cauchy-Kowalewski affirme qu' il existe une unique solution holomorphe au voisinage de $S$ dans $\boldsymbol{C}^{n+1}$. Est-ce que jusqu' où cette solution locale peut se prolonger analytiquement? Dans [H1], en utilisant les résultats de Bieberbach, Fatou et Picard, nous avons construit un exemple tel que le domaine d'holomorphie admette un point extérieur pour des opérateurs différentiels à coefficients de fonctions entières. Il s'agit alors des cas d'opérateurs différentiels de partie principale à coefficients polynomiaux.

Nous rappelons d'abord le résultat suivant.

THÉORÈME 1.1 [HLT]. Soit a $(x, D)$ un opérateur différentiel d'ordre $m$, holomorphe $\operatorname{sur}\left\{x ;\left|x_{0}\right| \leq R, x^{\prime} \in C^{n}\right\}$. Supposons que les coefficients de sa partie principale soient polynomiaux;

$$
\begin{aligned}
g(x, \xi) & =\xi_{0}^{m}+\sum_{k=1}^{m} L_{k}\left(x, \xi^{\prime}\right) \xi_{0}^{m-k}, \\
L_{k}\left(x, \xi^{\prime}\right) & =\sum_{\left|\alpha^{\prime}\right|=k,\left|\beta^{\prime}\right| \leq k \mu} a_{\alpha, \beta^{\prime}}\left(x_{0}\right) x^{\prime \beta^{\prime}} \xi^{\prime \alpha^{\prime}},
\end{aligned}
$$

$\mu$ étant un entier $(\geq 0)$, où les $a_{\alpha, \beta^{\prime}}\left(x_{0}\right)$ sont holomorphes sur $\left\{x_{0} ;\left|x_{0}\right| \leq R\right\}$. Posons $M(R)=\sup \left\{\left|a_{\alpha, \beta^{\prime}}\left(x_{0}\right)\right| ;\left|x_{0}\right| \leq R,|\alpha|=m,\left|\alpha^{\prime}\right|=k,\left|\beta^{\prime}\right| \leq k \mu, 1 \leq k \leq m\right\}$.

Il existe alors une constante $C(0<C \leq 1)$ ne dépendant que de $M(R)$ telle que la solution de (1.1) soit holomorphe sur $\left\{x ;\left|x_{0}\right| \leq C \min \left\{\left(1+\left\|x^{\prime}\right\|\right)^{-\mu_{0}}, R\right\}\right.$, où $\left\|x^{\prime}\right\|=$ $\max _{1 \leq i \leq n}\left|x_{i}\right|$ et $\mu_{0}=\max \{\mu-1,0\}$.

Nous rappelons que ce théorème résulte du lemme suivant.

LEMME 1.1 [HT]. Dans le problème (1.1), supposons que l'opérateur a et les données $v, w_{k}$ soient holomorphes sur $X=\left\{x ;\left|x_{j}\right| \leq r_{j}, 0 \leq j \leq n\right\}$, et notons $\left\|g(\cdot, \xi)-\xi_{0}^{m}\right\|_{X}$ une fonction spectrale de $g(x, D)-D_{0}^{m}$ sur ce domaine;

$$
H_{X}(\xi)=\left\|g(\cdot, \xi)-\xi_{0}^{m}\right\|_{X}=\sum_{|\alpha|=m, \alpha_{0}<m} \sup _{x \in X}\left|a_{\alpha}(x)\right| \xi^{\alpha}, \quad \xi \in\left(\boldsymbol{R}_{+}\right)^{n+1} .
$$

Choisissons un nombre $\theta_{0}\left(\geq 1 / r_{0}\right)$ satisfaisant

$$
H_{X}\left(1, \theta^{\prime} / \theta_{0}\right)<1 \quad \text { pour } \theta^{\prime}=\left(\theta_{1}, \ldots, \theta_{n}\right), \quad \theta_{j}=1 / r_{j}, \quad 1 \leq j \leq n .
$$

La solution $u(x)$ du problème (1.1) est alors holomorphe sur

$$
\left\{x ; \sum_{j=0}^{n} \theta_{j}\left|x_{j}\right|<1-H_{X}\left(1, \theta^{\prime} / \theta_{0}\right)\right\} \text {. }
$$

Note. La Proposition 8 dans [HLT] est d'une forme plus précise que ce lemme. 
Pour faciliter la preuve du Corollaire 1.2 ultérieurement, nous donnons ici une démonstration du Théorème 1.1.

Preuve du Théorème du 1.1. Posons

$$
H_{R, R_{1}}(\xi)=\left\|g(\cdot, \xi)-\xi_{0}^{m}\right\|_{X\left(R, R_{1}\right)}=\sum_{|\alpha|=m, \alpha_{0} \leq m-1} \sup _{x \in X\left(R, R_{1}\right)}\left|a_{\alpha}(x)\right| \xi^{\alpha},
$$

où $X\left(R, R_{1}\right)=\left\{x ;\left|x_{0}\right| \leq R,\left\|x^{\prime}\right\| \leq R_{1}\right\}$. On a, pour $\xi_{0} \geq 1 / R, \xi_{i}=1 / R_{1}, 1 \leq i \leq n$,

$$
\begin{aligned}
H_{R, R_{1}}\left(1, \xi^{\prime} / \xi_{0}\right) & \leq M(R) \sum_{k=1}^{m} \sup _{\left\|x^{\prime}\right\| \leq R_{1}}\left(1+\sum_{i=1}^{n}\left|x_{i}\right|\right)^{k \mu}\left(\sum_{i=1}^{n}\left|\xi_{i}\right| / \xi_{0}\right)^{k} \\
& \leq M(R) \sum_{k=1}^{m}\left[n\left(1+R_{1}\right)\right]^{k \mu}\left(n / R_{1} \xi_{0}\right)^{k} .
\end{aligned}
$$

Puisque $1 / R_{1} \leq 2 /\left(1+R_{1}\right),\left(R_{1} \geq 1\right)$, pour $\xi_{0} \geq \max \left\{2 n^{\mu+1}\left(1+R_{1}\right)^{\mu_{0}}, 1 / R\right\}, \xi_{i}=$ $1 / R_{1}, 1 \leq i \leq n, R_{1} \geq 1$, on a

$$
\begin{aligned}
H_{R, R_{1}}\left(1, \xi^{\prime} / \xi_{0}\right) & \leq M(R) \sum_{k=1}^{m}\left[n\left(1+R_{1}\right)\right]^{k \mu}\left[2 n /\left(1+R_{1}\right) \xi_{0}\right]^{k} \\
& \leq M(R) \sum_{k=1}^{m}\left[2 n^{\mu+1}\left(1+R_{1}\right)^{\mu_{0}} / \xi_{0}\right]^{k} \leq 2 m n^{\mu+1} M(R)\left(1+R_{1}\right)^{\mu_{0}} / \xi_{0} .
\end{aligned}
$$

Donc pour $\xi_{0} \geq \max \left\{4 m n^{\mu+1} M(R)\left(1+R_{1}\right)^{\mu_{0}}, 2 n^{\mu+1}\left(1+R_{1}\right)^{\mu_{0}}, 1 / R\right\}$, on a $H_{R, R_{1}}\left(1, \xi^{\prime} / \xi_{0}\right) \leq 1 / 2$.

D'après le lemme 1.1, la solution $u(x)$ du problème (1.1) est holomorphe sur $\left\{x ;\left|x_{0}\right| \leq\right.$ $\left.1 / 4(n+1) \xi_{0} \leq C_{1} \min \left[1 /\left(1+R_{1}\right)^{\mu_{0}}, R\right],\left\|x^{\prime}\right\| \leq C_{2} R_{1}\right\}$, pour $R_{1}(\geq 1)$ quelconque, $C_{1}, C_{2}$ $\left(0<C_{1} \leq 1, C_{2}>0\right)$ étant des constantes ne dépendant que de $M(R)$. Ceci démontre le Théorème 1.1.

Nous avons alors

Corollaire 1.1 [HLT]. Soit $\mathcal{D}$ un domaine de $\boldsymbol{C} . \mathcal{D} \ni 0$ est un point de base. $\mathcal{R}(\mathcal{D})$ est le revêtement universel de $\mathcal{D}$. Supposons que dans (1.1), les coefficients d'opérateur $a(x, D)$ et les données $v(x), w_{k}\left(x^{\prime}\right)$ se prolongent analytiquement $\operatorname{sur} \mathcal{R}(\mathcal{D}) \times \boldsymbol{C}^{n}$ et que la partie principale $g(x, D)$ holomorphe sur $\mathcal{R}(\mathcal{D}) \times \boldsymbol{C}^{n}$ satisfasse la condition du Théorème 1.1 avec $\mu=0,1$. Le problème (1.1) possède alors une unique solution holomorphe sur $\mathcal{R}(\mathcal{D}) \times \boldsymbol{C}^{n}$. En particulier, au cas de $\mathcal{D}=\boldsymbol{C}$, la solution est une fonction entière sur $\boldsymbol{C}^{n+1}$. Ceci a été déjà démontré dans $[\mathrm{P}],[\mathrm{WP}]$ et [HLT].

Note. Un résultat de [HLT] est d'une forme plus précise que ceci.

PREUVE. Soit $\gamma$ un chemin quelconque d'origine 0 dans $\mathcal{D} ; \gamma=\left\{x_{0}=\gamma(t) ; t \in\right.$ $[0,1]\}, \gamma(t)$ étant une fonction continue sur $[0,1]$. D'abord, notons que d'après le Théorème 1.1, la solution $u(x)$ est holomorphe sur $V(0) \times \boldsymbol{C}^{n}, V(0)$ étant un voisinage de 0 dans $\boldsymbol{C}$. Posons $\tau_{0}=\sup \tau$, où, pour $\gamma_{\tau}=\left\{x_{0}=\gamma(t) ; t \in[0, \tau], 0 \leq \tau \leq 1\right\}$, il existe un voisinage 
$V\left(\gamma_{\tau}\right)$ de $\gamma_{\tau}$ dans $C$ tel que la solution $u(x)$ du problème (1.1) soit holomorphe sur $V\left(\gamma_{\tau}\right) \times$ $\boldsymbol{C}^{n}$. Au voisinage de $x_{0}=\gamma\left(\tau_{0}\right)$, tous les coefficients $a_{\alpha, \beta^{\prime}}\left(x_{0}\right)$ en $x^{\prime}$ et $\xi$ de $g(x, \xi)$ sont holomorphes et bornés. Alors pour $\tau\left(0<\tau<\tau_{0}\right)$, près de $\tau_{0}$, les $D_{0}^{k} u\left(\gamma(\tau), x^{\prime}\right), 0 \leq$ $k \leq m-1$, sont holomorphes sur $\boldsymbol{C}^{n}$ et d'après le Théorème 1.1, $u(x)$ est holomorphe $\operatorname{sur} V\left(\gamma\left(\tau_{0}\right)\right) \times \boldsymbol{C}^{n}$. On voit donc que $\tau_{0}=1$ et que la solution $u(x)$ peut se prolonger analytiquement sur $V(\gamma) \times C^{n}, V(\gamma)$ étant un voisinage de $\gamma$ dans $C$. D'après les Propositions 7.1 et 7.2 dans [HLT], la solution $u(x)$ peut se prolonger analytiquement $\operatorname{sur} \mathcal{R}(\mathcal{D}) \times \boldsymbol{C}^{n}$. Ceci démontre le Corollaire 1.1.

Ce Corollaire se généralise comme suit.

Corollaire 1.2. Notons $z$ un point de $\boldsymbol{C}^{l}$. Soit a $\left(x, z, D_{x}, D_{z}\right)$ un opérateur différentiel holomorphe sur $\mathcal{R}(\mathcal{D}) \times C^{n} \times C^{l}$. Supposons que sa partie principale $g\left(x, D_{x}, D_{z}\right)$ soit indépendant de $z$ et de la forme

$$
g\left(x, D_{x}, D_{z}\right)=g_{0}\left(x, D_{x}\right)+g_{1}\left(x, D_{x}, D_{z}\right),
$$

où $g_{0}(x, \xi)$ est un polynôme en $\xi, x^{\prime}$ satisfaisant la condition du Théorème 1.1 avec $\mu=0,1$ et $g_{1}(x, \xi, \eta)=0$ pour $\eta=0$.

Considérons le problème de Cauchy

$$
\begin{gathered}
a\left(x, z, D_{x}, D_{z}\right) u(x, z)=v(x, z), \\
D_{0}^{k} u\left(0, x^{\prime}, z\right)=w_{k}\left(x^{\prime}, z\right), \quad 0 \leq k \leq m-1,
\end{gathered}
$$

où les $v(x, z), w_{k}\left(x^{\prime}, z\right)$ sont holomorphes sur $\mathcal{R}(\mathcal{D}) \times \boldsymbol{C}^{n} \times \boldsymbol{C}^{l}$. Ce problème (1.2) possède alors une unique solution holomorphe $\operatorname{sur} \mathcal{R}(\mathcal{D}) \times C^{n} \times C^{l}$.

Note. Ce n'est pas nécessaire que les coefficients de $g_{1}\left(x, D_{x}, D_{z}\right)$ sont polynomiaux en $x^{\prime}$.

Preuve. En utilisant le même raisonnement qu'au Corollaire 1.1, il suffit de démontrer ce Corollaire au cas où $\mathcal{D}=\left\{x_{0} \in \boldsymbol{C} ;\left|x_{0}\right| \leq R\right\}$, donc $\mathcal{R}(\mathcal{D})=\mathcal{D}$. Désignons par $H_{R, R_{1}}(\xi, \eta)=\left\|g(\cdot, \xi, \eta)-\xi_{0}^{m}\right\|_{X\left(R, R_{1}\right)}$ une fonction spectrale de

$$
\begin{gathered}
g\left(x, D_{x}, D_{z}\right)-D_{0}^{m} \quad \operatorname{sur} \quad X\left(R, R_{1}\right)=\left\{(x, z) ;\left|x_{0}\right| \leq R,\left\|x^{\prime}\right\| \leq R_{1}\right\} ; \\
H_{R, R_{1}}(\xi, \eta)=\sum_{|\alpha|+|\beta|=m, \alpha_{0} \leq m-1} \sup _{x \in X\left(R, R_{1}\right)}\left|a_{\alpha, \beta}(x)\right| \xi^{\alpha} \eta^{\beta}
\end{gathered}
$$

pour $g(x, \xi, \eta)=\sum_{|\alpha|+|\beta|=m} a_{\alpha, \beta}(x) \xi^{\alpha} \eta^{\beta}$. On a alors

$$
H_{R, R_{1}}(\xi, \eta)=H_{R, R_{1}}^{0}(\xi)+H_{R, R_{1}}^{1}(\xi, \eta),
$$

où $H_{R, R_{1}}^{0}(\xi)$ et $H_{R, R_{1}}^{1}(\xi, \eta)$ sont des fonctions spectrales de

$$
g_{0}\left(x, D_{x}\right)-D_{0}^{m}=\sum_{k=1}^{m}\left(\sum_{\left|\alpha^{\prime}\right|=k,\left|\beta^{\prime}\right| \leq k \mu} a_{\alpha, \beta^{\prime}}^{0}\left(x_{0}\right) x^{\prime \beta^{\prime}} D_{x^{\prime}}^{\alpha^{\prime}}\right) D_{0}^{m-k},
$$

avec $\mu=0,1$, et

$$
g_{1}\left(x, D_{x}, D_{z}\right)=\sum_{|\alpha|+|\beta|=m,|\beta| \geq 1} a_{\alpha, \beta}^{1}(x) D_{x}^{\alpha} D_{z}^{\beta},
$$


respectivement. Pour $\xi_{0} \geq n(n+1), \xi_{i}=1 / R_{1}, 1 \leq i \leq n, R_{1} \geq n+l$, on a

$$
\begin{aligned}
H_{R, R_{1}}^{0}\left(1, \xi^{\prime} / \xi_{0}\right) & \leq M_{0}(R) \sum_{k=1}^{m}\left(1+n R_{1}\right)^{k}\left(n\left\|\xi^{\prime}\right\| / \xi_{0}\right)^{k}=M_{0}(R) \sum_{k=1}^{m}\left(1+n R_{1}\right)^{k}\left(n / R_{1} \xi_{0}\right)^{k} \\
& \leq M_{0}(R) \sum_{k=1}^{m}\left[(n+1) n / \xi_{0}\right]^{k} \leq m n(n+1) M_{0}(R) / \xi_{0},
\end{aligned}
$$

où $M_{0}(R)=\sup _{\left|x_{0}\right| \leq R, \alpha, \beta^{\prime}}\left|a_{\alpha, \beta^{\prime}}^{0}\left(x_{0}\right)\right|$, et pour $\left\|\xi^{\prime}\right\| \geq\|\eta\|$,

$$
\begin{aligned}
H_{R, R_{1}}^{1}\left(1, \xi^{\prime} / \xi_{0}, \eta / \xi_{0}\right) & \leq M_{1}\left(R, R_{1}\right) \sum_{k=1}^{m}\left(n\left\|\xi^{\prime}\right\|+l\|\eta\|\right)^{k-1}\|\eta\| / \xi_{0}^{k} \\
& \leq M_{1}\left(R, R_{1}\right) \sum_{k=1}^{m}\left[(n+l)\left\|\xi^{\prime}\right\|\right]^{k-1}\|\eta\| \leq m M_{1}\left(R, R_{1}\right)\|\eta\|,
\end{aligned}
$$

où $M_{1}\left(R, R_{1}\right)=\sup _{x \in X\left(R, R_{1}\right), \alpha, \beta}\left|a_{\alpha, \beta}^{1}(x)\right|$. Par conséquent, pour $\xi_{0} \geq \max \{4 m n(n+$ 1) $\left.M_{0}(R), n(n+1), 1 / R\right\}, \xi_{i}=1 / R_{1}, 1 \leq i \leq n, R_{1} \geq n+l, \eta_{j}=1 / R_{2}, 1 \leq j \leq l$, $R_{2} \geq \max \left\{4 m M_{1}\left(R, R_{1}\right), R_{1}\right\}$, on obtient $H_{R, R_{1}}^{0}\left(1, \xi^{\prime} / \xi_{0}\right), H_{R, R_{1}}^{1}\left(1, \xi^{\prime} / \xi_{0}, \eta / \xi_{0}\right) \leq 1 / 4$, et donc $H_{R, R_{1}}\left(1, \xi^{\prime} / \xi_{0}, \eta / \xi_{0}\right) \leq 1 / 2$.

D'après le lemme 1.1 , la solution $u(x, z)$ est alors holomorphe sur

$$
\left\{(x, z) ;\left|x_{0}\right| \leq 1 / 4(n+l+1) \xi_{0},\left\|x^{\prime}\right\| \leq 1 / 4(n+l+1)\left\|\xi^{\prime}\right\|,\|z\| \leq 1 / 4(n+l+1)\|\eta\|\right\},
$$

par suite, pour $R_{1}(\geq n+l), R_{2}\left(\geq \max \left\{4 m M_{1}\left(R, R_{1}\right), R_{1}\right\}\right)$ quelconques, la $u(x, z)$ est holomorphe $\operatorname{sur}\left\{(x, z) ;\left|x_{0}\right| \leq C_{0} \min [1, R],\left\|x^{\prime}\right\| \leq C_{1} R_{1},\|z\| \leq C_{2} R_{2}\right\}, C_{0}\left(0<C_{0} \leq\right.$ $1), C_{i}(>0), i=1,2$, étant des constantes ne dépendant que de $M_{0}(R)$. La solution $u(x, z)$ est donc holomorphe $\operatorname{sur}\left\{(x, z) ;\left|x_{0}\right| \leq C_{0} \min [1, R],\left(x^{\prime}, z\right) \in \boldsymbol{C}^{n} \times \boldsymbol{C}^{l}\right\}$. Comme dans le Corollaire 1.1, ceci démontre le Corollaire 1.2.

Dans [H2], [H3], en utilisant les résultats de fonction modulaire et son équation différentielle, et des équations différentielles de Darboux-Halphen et de Chazy [C], nous avons donné des exemples tels que le domaine d'holomorphie de la solution ait l'extérieur non vide pour les opérateurs différentiels à coefficients polynomiaux. Dans ces exemples, la dimension $n$ de l'espace $x^{\prime}=\left(x_{1}, \ldots, x_{n}\right)$ est toujours $n \geq 2$. Ferruccio Colombini nous a demandé si, au cas de $n=1$, le domaine d'holomorphie de la solution peut avoir l'extérieur non vide ou non. Dans cet article, nous observons le cas de $n=1$, en donnant quelques exemples pour certains opérateurs différentiels de partie principale à coefficients polynomiaux.

2. Quelques exemples. Étudions le problème de Cauchy

$$
\begin{gathered}
\left(D_{0}^{m}-\alpha x_{0}^{p} x_{1}^{q} D_{1}^{m}\right) u(x)=b(x, D) u(x)+v(x), \\
D_{0}^{k} u\left(0, x_{1}\right)=w_{k}\left(x_{1}\right), \quad 0 \leq k \leq m-1, \quad x=\left(x_{0}, x_{1}\right) \in C^{2},
\end{gathered}
$$

où $\alpha$ est une constante, $p(\geq 0), q(\geq m+1)$ sont des entiers et $b(x, D)$ est un opérateur d'ordre $m-1$. Les coefficients de $b(x, D), v(x)$ et $w_{k}\left(x_{1}\right), 0 \leq k \leq m-1$, sont holomorphes $\operatorname{sur} C^{2}$. 
Nous avons alors

Proposition 2.1. Le problème de Cauchy (2.1) possède une unique solution holomorphe sur $\mathcal{R}\left[\boldsymbol{C}^{2} \backslash \bigcup_{i=0}^{r} K_{i}\right]$, où $K_{i}, 1 \leq i \leq r$, sont les composantes irréductibles de $\left\{x \in \boldsymbol{C}^{2} ; \alpha \lambda^{m} x_{0}^{p+m} x_{1}^{q-m}=1\right\}, r$ étant un entier $(\geq 1), K_{0}=\left\{x \in \boldsymbol{C}^{2} ; x_{1}=0\right\}$ et les $K_{i}, 0 \leq i \leq r$, sont des surfaces caractéristiques pour $D_{0}^{m}-\alpha x_{0}^{p} x_{1}^{q} D_{1}^{m}$.

Note. Au cas de $0 \leq q \leq m$, ou bien $\alpha=0$ dans le problème (2.1), vu le Corollaire 1.1, la solution est holomorphe sur $\boldsymbol{C}^{2}$.

PREUVE. En effectuant le changement de variable $X_{1}=1 / x_{1}$, le problème (2.1) se transforme en le problème

$$
\begin{gathered}
{\left[D_{0}^{m}-\alpha x_{0}^{p} X_{1}^{-q}\left(-X_{1}^{2} D_{X_{1}}\right)^{m}\right] U\left(x_{0}, X_{1}\right)} \\
=b\left(x_{0}, 1 / X_{1}, D_{0},-X_{1}^{2} D_{X 1}\right) U\left(x_{0}, X_{1}\right)+v\left(x_{0}, 1 / X_{1}\right), \\
\quad D_{0}^{k} U\left(0, X_{1}\right)=w_{k}\left(1 / X_{1}\right), \quad 0 \leq k \leq m-1,
\end{gathered}
$$

où $U\left(x_{0}, X_{1}\right)=u\left(x_{0}, 1 / X_{1}\right)$. En plus, faisons le changement de variables au voisinage d'un point $\left(x_{0}, X_{1}\right)=\left(0, X_{1}^{(0)}\right), X_{1}^{(0)} \neq 0$, en fixant une détermination de $X_{1}^{\lambda}$ au point $X_{1}^{(0)}$, si $\lambda$ est un nombre rationnel;

$$
t=x_{0} / X_{1}^{\lambda}, \quad s=X_{1}, \quad \lambda=(q-m) /(p+m) .
$$

On a alors

$$
D_{0}=s^{-\lambda} D_{t}, \quad D_{X_{1}}=(1 / s)\left(-\lambda t D_{t}+s D_{s}\right) .
$$

Le problème (2.2) se transforme alors en le problème

$$
\begin{gathered}
{\left[D_{t}^{m}+G\left(t, D_{t}, s D_{s}\right)\right] \tilde{U}(t, s)=B\left(t, s, D_{t}, D_{s}\right) \tilde{U}(t, s)+\tilde{V}(t, s),} \\
D_{t}^{k} \tilde{U}(0, s)=\tilde{W}_{k}(s), \quad 0 \leq k \leq m-1,
\end{gathered}
$$

où $G\left(t, \eta_{0}, \eta_{1}\right), B\left(t, s, \eta_{0}, \eta_{1}\right)$ sont des polynômes de degrés respectifs $m$ et $m-1$ en $\eta_{0}, \eta_{1}$ à coefficients se prolongeant analytiquement $\operatorname{sur} \mathcal{R}\left[t \in \boldsymbol{C} ; \alpha \lambda^{m} t^{p+m} \neq 1\right] \times \mathcal{R}[s \in \boldsymbol{C} ; s \neq 0]$, et $G\left(t, \eta_{0}, \eta_{1}\right)$ ne contient pas $\eta_{0}^{m}$. En posant $s=e^{y}$, le problème (2.5) se transforme en le problème

$$
\begin{gathered}
{\left[D_{t}^{m}+G\left(t, D_{t}, D_{y}\right)\right] \hat{U}(t, y)=\hat{B}\left(t, y, D_{t}, D_{y}\right) \hat{U}(t, y)+\hat{V}(t, y),} \\
D_{t}^{k} \hat{U}(0, y)=\hat{W}_{k}(y), \quad 0 \leq k \leq m-1,
\end{gathered}
$$

où $\hat{B}$ est un opérateur différentiel d'ordre $m-1$, et ses coefficients, $\hat{V}(t, y)$ et $\hat{W}_{k}(y)$ sont des fonctions holomorphes sur $\mathcal{R}\left[t \in \boldsymbol{C} ; \alpha \lambda^{m} t^{p+m} \neq 1\right] \times\{y \in \boldsymbol{C}\}$.

D'après le Corollaire 1.1, la solution $\hat{U}(t, y)$ de (2.6) se prolonge analytiquement sur $\mathcal{R}\left[t \in \boldsymbol{C} ; \alpha \lambda^{m} t^{p+m} \neq 1\right] \times\{y \in \boldsymbol{C}\}$. Soient $\tilde{K}_{i}(1 \leq i \leq r)$, les composantes irréductibles de $\left\{\left(x_{0}, X_{1}\right) \in C^{2} ; X_{1}^{q-m}=\alpha \lambda^{m} x_{0}^{p+m}\right\}$ et $\tilde{K}_{0}=\left\{\left(x_{0}, X_{1}\right) \in \boldsymbol{C}^{2} ; X_{1}=0\right\}$. La solution $U\left(x_{0}, X_{1}\right)$ de (2.2) se prolonge analytiquement sur $\mathcal{R}\left[\boldsymbol{C}^{2} \backslash \bigcup_{i=0}^{r} \tilde{K}_{i}\right]$, donc la solution $u(x)$ du problème (2.1) se prolonge analytiquement sur $\mathcal{R}\left[\boldsymbol{C}^{2} \backslash \bigcup_{i=0}^{r} K_{i}\right]$. Ceci démontre la Proposition 2.1 . 
Nous donnons la Remarque suivante sur les singularités sur $K_{0}$ de la solution du problème de (2.1) dans la Proposition 2.1.

REMARQUE 2.1. Considérons le problème de Cauchy

$$
\left(D_{0}+x_{1}^{2} D_{1}\right) u(x)=x_{0} x_{1}, \quad u\left(0, x_{1}\right)=0 .
$$

La solution est

$$
u(x)=-x_{0}+\frac{1+x_{0} x_{1}}{x_{1}} \log \left(1+x_{0} x_{1}\right)
$$

qui peut se prolonger analytiquement sur $\mathcal{R}\left[C^{2} \backslash\left(K_{0} \cup K_{1}\right)\right]$, où $K_{1}=\left\{x ; 1+x_{0} x_{1}=\right.$ $0\}, K_{0}=\left\{x ; x_{1}=0\right\}$.

En utilisant le Corollaire 1.2, ce résultat se généralise facilement comme suit. Considérons le problème de Cauchy

$$
\begin{gathered}
{\left[D_{0}^{m}-\alpha x_{0}^{p} x_{1}^{q} D_{1}^{m}+h\left(x_{0}, x_{1}, D\right)\right] u(x)=b(x, D) u(x)+v(x),} \\
D_{0}^{k} u\left(0, x^{\prime}\right)=w_{k}\left(x^{\prime}\right), \quad 0 \leq k \leq m-1, \\
x=\left(x_{0}, x^{\prime}\right), \quad x^{\prime}=\left(x_{1}, x^{\prime \prime}\right), \quad x^{\prime \prime}=\left(x_{2}, \ldots, x_{n}\right) \in C^{n-1},
\end{gathered}
$$

où $h\left(x_{0}, x_{1}, D\right), b(x, D)$ sont des opérateurs d'ordres respectifs $m$ et $m-1$, holomorphes sur $C^{n+1}$, et $h\left(x_{0}, x_{1}, \xi_{0}, \xi_{1}, \xi^{\prime \prime}\right)=0$, pour $\xi^{\prime \prime}=\left(\xi_{2}, \cdot, \cdot, \cdot, \xi_{n}\right)=0 . v(x), w_{k}\left(x^{\prime}\right)$ sont des fonctions holomorphes sur $\boldsymbol{C}^{n+1}$.

Nous avons alors

COROLlaire 2.1. Le problème de Cauchy (2.7) possède une unique solution holomorphe sur $\mathcal{R}\left[\boldsymbol{C}^{2} \backslash \bigcup_{i=1}^{r} K_{i}\right] \times \boldsymbol{C}^{n-1}$, où les $K_{i}, 1 \leq i \leq r$, sont des surfaces caractéristiques dans la Proposition 2.1.

Cette méthode est applicable aux problèmes de Cauchy suivants. Considérons le problème

$$
\begin{gathered}
\left(D_{0}-2 x_{0} x_{1}^{2} D_{1}\right) D_{0} u(x)=b(x, D) u(x)+v(x), \\
D_{0}^{k} u\left(0, x_{1}\right)=w_{k}\left(x_{1}\right), \quad 0 \leq k \leq 1, \quad x=\left(x_{0}, x_{1}\right) \in C^{2},
\end{gathered}
$$

où le $b(x, D)$ est d'ordre 1 , et ses coefficients, $v(x)$ et $w_{k}\left(x_{1}\right)$ sont holomorphes sur $C^{2}$. La solution de (2.8) est holomorphe sur $\mathcal{R}\left[\boldsymbol{C}^{2} \backslash\left(K_{0} \cup K_{1}\right)\right], K_{1}=\left\{x \in \boldsymbol{C}^{2} ; x_{0}^{2} x_{1}=1\right\}$, $K_{0}=\left\{x \in C^{2} ; x_{1}=0\right\}$. Ensuite, considérons le problème pour un opérateur d'un type de Clairaut $(m \geq 2)$

$$
\begin{gathered}
{\left[D_{0}^{m}+\left(x_{0} D_{0}-x_{1} D_{1}\right)\left(-x_{1}^{2} D_{1}\right)^{m-1}\right] u(x)=b(x, D) u(x)+v(x),} \\
D_{0}^{k} u\left(0, x_{1}\right)=w_{k}\left(x_{1}\right), \quad 0 \leq k \leq m-1, \quad x=\left(x_{0}, x_{1}\right) \in C^{2},
\end{gathered}
$$

où le $b(x, D)$ est d'ordre $m-1$ et ses coefficients, $v(x)$ et $w_{k}\left(x_{1}\right)$ sont holomorphes sur $C^{2}$. Par le changement de variable $X_{1}=1 / x_{1}$, le problème (2.9) se transforme en le problème de 
Cauchy

$$
\begin{gathered}
{\left[D_{0}^{m}+\left(x_{0} D_{0}+X_{1} D_{X_{1}}\right) D_{X_{1}}^{m-1}\right] U\left(x_{0}, X_{1}\right)} \\
\quad=\tilde{b}\left(x_{0}, X_{1}, D_{0}, D_{X_{1}}\right) U\left(x_{0}, X_{1}\right)+\tilde{v}\left(x_{0}, X_{1}\right), \\
D_{0}^{k} U\left(0, X_{1}\right)=\tilde{w}_{k}\left(X_{1}\right), \quad 0 \leq k \leq m-1,
\end{gathered}
$$

où le $\tilde{b}$ est d'ordre $m-1$ et ses coefficients, $\tilde{v}\left(x_{0}, X_{1}\right)$ et $\tilde{w}_{k}\left(X_{1}\right)$ sont holomorphes sur $C^{2} \backslash\left\{X_{1}=0\right\}$. La partie principale de l'opérateur de (2.10) est un opérateur particulier dans [D]. L'équation de courbe bicaractéristique est

$$
X_{1}=x_{0} \frac{d X_{1}}{d x_{0}}+(-1)^{m-1}\left(\frac{d X_{1}}{d x_{0}}\right)^{m},
$$

qui est une équation différentielle d'un type de Clairaut. En effectuant le changement de variables

$$
t=x_{0} / X_{1}^{(m-1) / m}, \quad s=X_{1}
$$

et en utilisant une méthode de cette section, on voit que la solution de (2.9) est holomorphe $\operatorname{sur} \mathcal{R}\left[\boldsymbol{C}^{2} \backslash\left(K_{0} \cup K_{1}\right)\right], K_{1}=\left\{x \in \boldsymbol{C}^{2} ; 1-(-1)^{m}\left((m-1)^{m-1} / m^{m}\right) x_{0}^{m} x_{1}^{m-1}=0\right\}, K_{0}=$ $\left\{x \in C^{2} ; x_{1}=0\right\}$. Le raisonnement de la Proposition 2.1 s'applique à un problème du type suivant dont un cas particulier est posé par Tatsuya Koike. En fait, dans [K], il a posé une question sur des singularités d'une solution d'équation

$$
\left(\frac{\partial^{2}}{\partial x^{2}}-\frac{1}{x} \frac{\partial^{2}}{\partial y^{2}}-\frac{c}{x^{2}} \frac{\partial}{\partial y}\right) u(x, y)=0,
$$

$c$ étant une constante. En posant $x_{0}=y, x_{1}=x$ dans le Corollaire suivant, on obtient une solution de cette équation.

\section{COROllaire 2.2. Considérons le problème de Cauchy}

$$
\begin{gathered}
\left(D_{0}^{m}-\alpha x_{0}^{p} x_{1}^{q} D_{1}^{m}\right) u(x)=b(x, D) u(x)+v(x), \\
D_{0}^{k} u\left(0, x^{\prime}\right)=w_{k}\left(x^{\prime}\right), \quad 0 \leq k \leq m-1, \quad x=\left(x_{0}, x^{\prime}\right) \in C^{n+1},
\end{gathered}
$$

où $\alpha$ est une constante, $p(\geq 0), q$ sont des entiers et $b(x, D)$ est un opérateur d'ordre $m-1$. Les coefficients de $b(x, D), v(x)$ et $w_{k}\left(x^{\prime}\right), 0 \leq k \leq m-1$, sont holomorphes sur $\mathcal{R}\left[C^{n+1} \backslash\right.$ $\left.\left\{x_{1}=0\right\}\right]$. Le problème de Cauchy (2.1) possède alors une unique solution holomorphe sur $\mathcal{R}\left[\boldsymbol{C}^{n+1} \backslash \bigcup_{i=0}^{r} K_{i}\right]$, où $K_{0}=\left\{x \in \boldsymbol{C}^{n+1} ; x_{1}=0\right\}$ et $K_{i}, 1 \leq i \leq r$, sont les composantes irréductibles de $\left\{\left(x_{0}, x_{1}\right) \in C^{n+1} ; x_{1}^{m-q}=\alpha \mu^{m} x_{0}^{p+m}\right\}, \mu=(m-q) /(p+m)$, r étant un entier $(\geq 1) . K_{i}, 0 \leq i \leq r$, sont des surfaces caractéristiques pour $D_{0}^{m}-\alpha x_{0}^{p} x_{1}^{q} D_{1}^{m}$, issues de $S \cap\left\{x_{1}=0\right\}$.

Note. Ce n'est pas nécessaire que $q$ est un entier $(\geq 0)$.

Preuve. En effet, le changement de variables $t=x_{0} / x_{1}^{\mu}, x_{1}=s=e^{y}$, et le raisonnement de la Proposition 2.1 prouve immédiatement ce Corollaire.

Bien entendu, de même, pour un type de Clairaut, on a 
COROllaire 2.3. Considérons le problème de Cauchy $(m \geq 2)$

$$
\begin{gathered}
{\left[D_{0}^{m}+\left(x_{0} D_{0}+x_{1} D_{1}\right) D_{1}^{m-1}\right] u(x)=b(x, D) u(x)+v(x),} \\
D_{0}^{k} u\left(0, x^{\prime}\right)=w_{k}\left(x^{\prime}\right), \quad 0 \leq k \leq m-1, \quad x=\left(x_{0}, x^{\prime}\right) \in C^{n+1},
\end{gathered}
$$

où le b est d'ordre $m-1$ et ses coefficients, $v(x)$ et $w_{k}\left(x^{\prime}\right)$ sont holomorphes sur $\mathcal{R}\left[C^{n+1} \backslash\right.$ $\left.\left\{x_{1}=0\right\}\right]$. La solution de (2.12) est holomorphe sur $\mathcal{R}\left[\boldsymbol{C}^{n+1} \backslash\left\{K_{0} \cup K_{1}\right\}\right]$, où $K_{0}=\left\{x ; x_{1}=\right.$ $0\}$ et $K_{1}=\left\{x \in C^{n+1} ; x_{1}^{m-1}-(-1)^{m}\left((m-1)^{m-1} / m^{m}\right) x_{0}=0\right\}$.

\section{RÉFÉRENCES}

[C] J. CHAZY, Sur les équations différentielles du troisième ordre et d'ordre supérieur dont l'intégrale générale a ses points critiques fixes, Acta Math. 34 (1911), 317-385.

[D] S. Delache, Les solutions élémentaires hyperboliques d'opérateurs de Tricomi-Clairaut, Bull. Soc. Math. France 97 (1969), 5-79.

[F] R. FORSYTH, Theory of differential equations, Dover, 1958.

[GKL] L. GÅrding, T. Kotake et J. Leray, Uniformisation et développement asymptotique de la solution du problème de Cauchy linéaire à données holomorphes; analogue avec la théorie des ondes asymptotiques et approchées, Bull. Soc. Math. France 92 (1964), 263-361.

[HLT] Y. Hamada, J. Leray et A. TAKeuchi, Prolongements analytiques de la solution du problème de Cauchy linéaire, J. Math. Pures Appl. 64 (1985), 257-319.

[H1] Y. HAMADA, Une remarque sur le domaine d'existence de la solution du problème de Cauchy pour l'opérateur différentiel à coefficients des fonctions entières, Tôhoku Math. J. 50 (1998), 133-138.

[H2] Y. HAMADA, Une remarque sur le problème de Cauchy pour l'opérateur différentiel de partie principale à coefficients polynomiaux, Tohoku Math. J. 52 (2000), 79-94.

[H3] Y. HAmADA, Une remarque sur le problème de Cauchy pour l'opérateur différentiel de partie principale à coefficients polynomiaux II, Tohoku Math. J. 54 (2002), 297-307.

[HT] Y. Hamada ET A. TAKeUChI, Sur le prolongement analytique de la solution du problème de Cauchy, C.R. Acad. Sc. Paris Sér. I Math. 295, (1982), 329-332.

[Hi] E. Hille, Ordinary Differential Equations in the Complex Domain, John Wiley, New York-London-Sydney, 1976.

[K] T. KoIKE, Un exposé oral dans le séminaire du 29 Janv. 2002, à l'Univ. de Kyoto.

[L] J. LERAY, Uniformisation de la solution du problème linéaire analytique de Cauchy près de la variété qui porte les données de Cauchy (Problème de Cauchy I), Bull. Soc. Math. France 85 (1957), 389-429.

[N] T. Nishino, Theory of Functions of Several Complex Variables [Tahensu Kansu Ron] (en japonais), Univ. of Tokyo Press, 1996.

[P] J. PERSSON, On the local and global non-characteristic Cauchy problem when the solutions are holomorphic functions or analytic functionals in the space variables, Ark. Mat. 9 (1971), 171-180.

[PW] P. Pongérard ET C. Wagschal, Problème de Cauchy dans des espaces de fonctions entières, J. Math. Pures Appl. 75 (1996), 409-418.

61-36 TATEKURA-CHO, SHIMOgAmo

SAKYO-KU, KYOTO, 606-0806

JAPAN

E-mail address: yhamada@oak.ocn.ne.jp 